The Astrophysical JouRnal, 559:147-156, 2001 September 20

(C) 2001. The American Astronomical Society. All rights reserved. Printed in U.S.A.

\title{
CIRCUMNUCLEAR STELLAR POPULATION, MORPHOLOGY, AND ENVIRONMENT OF SEYFERT 2 GALAXIES: AN EVOLUTIONARY SCENARIO
}

\author{
Thaisa Storchi-BergmanN \\ Instituto de Física, Universidade Federal do Rio Grande Sol, Campus do Vale, CP 15051, Porto Alegre, Brasil; thaisa@if.ufrgs.br \\ Rosa M. GonzÁlez Delgado \\ Instituto de Astrofísica de Andalucía (CSIC), Apartado Correos 3004, Granada 18080, Spain; rosa@iaa.es \\ HENRIQUE R. SCHMITT ${ }^{1}$ \\ National Radio Astronomy Observatories, P.O. Box O, Socorro, NM 87801; hschmitt@aoc.nrao.edu \\ R. Cid FERNANDES ${ }^{2}$ \\ Department of Physics and Astronomy, Johns Hopkins University, Baltimore, MD 21218; cid@pha.jhu.edu \\ AND \\ TIMOTHY HECKMAN \\ Department of Physics and Astronomy, Johns Hopkins University, Baltimore, MD 21218; heckman@pha.jhu.edu \\ Received 2001 February 14; accepted 2001 May 10
}

\begin{abstract}
We investigate the relation between the characteristics of the circumnuclear stellar population and both the galaxy morphology and the presence of close companions for a sample of 35 Seyfert 2 nuclei. Fifteen galaxies present unambiguous signatures of recent episodes of star formation within $\approx 300 \mathrm{pc}$ of the nucleus. When we relate this property to the Hubble type of the host galaxy, we find that the incidence of recent circumnuclear star formation increases along the Hubble sequence; it seems to be greater than that in non-Seyfert galaxies for the early Hubble types S0 and Sa but similar to that in non-Seyfert galaxies for later Hubble types. In both early-type and late-type Seyfert galaxies, the presence of recent circumnuclear star formation is related to the galaxy morphology in the inner few kiloparsecs, as observed in Hubble Space Telescope images through the filter F606W by Malkan et al., who have assigned a late "inner Hubble type" to most Seyfert 2 galaxies with recent circumnuclear star formation. This new classification is due to the presence of dust lanes and spiral structures in the inner region. The presence of recent star formation around Seyfert 2 nuclei is also related to interactions: among the 13 galaxies of the sample with close companions or in mergers, nine have recent star formation in the nuclear region. These correlations between the presence of companions, the inner morphology, and the incidence of recent star formation suggest an evolutionary scenario in which the interaction is responsible for sending gas inward, which both feeds the active galactic nucleus and triggers star formation. The starburst then fades with time and the composite Seyfert $2+$ starburst nucleus evolves to a "pure "Seyfert 2 nucleus with an old stellar population. This scenario can reconcile the hypothesis that the active nucleus in Seyfert galaxies is triggered by interactions with the results of previous studies, which find only a small excess of interacting galaxies in Seyfert samples when compared with non-Seyfert samples. The large excess can only be found early after the interaction, in the phase in which a composite (Seyfert + starburst) nucleus is observed.

Subject headings: galaxies: active — galaxies: nuclei — galaxies: Seyfert — galaxies: starburst — galaxies: stellar content
\end{abstract}

\section{INTRODUCTION}

The recent evidence for a proportionality between galactic bulges and nuclear black hole masses (Magorrian et al. 1998; Ferrarese \& Merrit 2000; Gebhart et al. 2000) and the fact that supermassive black holes seem to be present in the nuclei of most present-day galaxies (Ho 1999) point to a "starburst-active galactic nucleus (AGN) connection" operating at the epoch of galaxy formation. Circumstantial evidence for this connection is found in, e.g., the quasar host images obtained with the Hubble Space Telescope (HST; Bahcall et al. 1997) that show distorted morphologies due to interactions, characteristic of luminous starbursts in the near universe, and ultraluminous infrared galaxies (ULIRGs) with quasar luminosities $\left(L_{\mathrm{IR}}>10^{12} L_{\odot}\right)$. The latter are in most cases star-forming merger systems, argued

\footnotetext{
${ }^{1}$ Jansky Fellow.

${ }^{2}$ Gemini Fellow.
}

to be the initial dust-enshrouded stage of a quasar. Spectral signatures of an aging starburst have indeed been found in a few quasi-stellar objects (QSOs) and ULIRGs (Brotherton et al. 1999; Canalizo \& Stockton 2000), interpreted as being objects in the transition phase between the starburst and the QSO.

In the near universe, starburst galaxies and active nuclei share a fundamental characteristic: both are dependent on gas to fuel the birth of new stars in the first case and to feed the nuclear black hole in the second. If there is a gas flow to the center, it may trigger star formation. This is the essence of the hybrid models proposed by Perry \& Dyson (1985) and Norman \& Scoville (1988). More recently, Collin \& Zahn (1999) argued that star formation events can occur as far inward as in the outskirts of the accretion disk where the gas is gravitationally unstable.

Observational evidence for nuclear starbursts around nearby AGNs includes the works of Terlevich, Diaz, \& Ter- 
TABLE 1

SAMPLE Properties

\begin{tabular}{|c|c|c|c|c|c|c|}
\hline \multirow[b]{2}{*}{ GaLAXY } & \multirow[b]{2}{*}{$\begin{array}{l}V_{\mathrm{GSR}} \\
(1)\end{array}$} & \multirow[b]{2}{*}{$\begin{array}{l}M_{B_{T}^{o}} \\
(2)\end{array}$} & \multirow{2}{*}{$\begin{array}{c}\text { SCALE } \\
\left(\mathrm{pc} \operatorname{arcsec}^{-1}\right) \\
(3)\end{array}$} & \multicolumn{2}{|c|}{ Hubble TyPe } & \multirow[b]{2}{*}{$\begin{array}{c}\text { POPULATION }^{\mathrm{a}} \\
\text { (6) }\end{array}$} \\
\hline & & & & $\begin{array}{l}\mathrm{RC} 3 \\
(4)\end{array}$ & $\begin{array}{l}\text { MGT } \\
(5)\end{array}$ & \\
\hline \multicolumn{7}{|c|}{ Southern Sample } \\
\hline NGC $1358 \ldots \ldots \ldots \ldots$ & 3980 & -20.92 & 257.3 & $\mathrm{SB} 0 / \mathrm{a}$ & SB0 & 2 \\
\hline NGC 1386 .............. & 741 & -19.02 & $81.9^{\mathrm{b}}$ & SB0 & $\mathrm{Sb} / \mathrm{c}$ & 2 \\
\hline NGC 3081 .............. & 2164 & -19.71 & 139.9 & SB0/a & $\mathrm{SB} 0 / \mathrm{a}$ & 3 \\
\hline NGC $5135 \ldots \ldots \ldots \ldots . . .$. & 3959 & -21.24 & 255.9 & SBab & Sc & 1 \\
\hline NGC 5643 .............. & 1066 & -20.53 & 68.91 & $\mathrm{SABc}$ & $\ldots$ & 1 \\
\hline NGC $6300 \ldots \ldots \ldots \ldots . . . .$. & 997 & -20.42 & 64.45 & $\mathrm{SBb}$ & Sc & 2 \\
\hline NGC $6890 \ldots \ldots \ldots \ldots . . . .$. & 2459 & -19.76 & 158.9 & $\mathrm{Sb}$ & $\ldots$ & 3 \\
\hline NGC $7130 \ldots \ldots \ldots \ldots$ & 4850 & -21.17 & 313.5 & $\mathrm{Sa}$ & $\mathrm{Sd}$ & 1 \\
\hline NGC $7582 \ldots \ldots \ldots \ldots$ & 1551 & -20.75 & 100.3 & $\mathrm{SBab}$ & $?$ & 1 \\
\hline Mrk $348 \ldots \ldots \ldots \ldots \ldots$ & 4669 & -20.03 & 301.8 & $\mathrm{~S} 0 / \mathrm{a}$ & S0 & 3 \\
\hline Mrk $573 \ldots \ldots \ldots \ldots \ldots$ & 5161 & -20.62 & 333.6 & SB0 & S0 & 3 \\
\hline Mrk $607 \ldots \ldots \ldots \ldots \ldots$ & 2716 & -19.48 & 175.6 & $\mathrm{Sa}$ & $\mathrm{Sb}$ & 2 \\
\hline Mrk $1210 \ldots \ldots \ldots \ldots \ldots$ & 3910 & -19.38 & 252.7 & $\mathrm{Sa}^{\mathrm{c}}$ & $\mathrm{Sa}$ & 1 \\
\hline CGCG 420-015 ......... & 8811 & -20.35 & 569.5 & $\mathrm{Sa}^{\mathrm{c}}$ & $\mathrm{Sa}$ & 2 \\
\hline IC $1816 \ldots \ldots \ldots \ldots \ldots$ & 5086 & -20.5 & 328.8 & $\mathrm{Sab}$ & $\mathrm{SBa} / \mathrm{b}$ & 3 \\
\hline IRAS $11215-2806 \ldots \ldots$ & 4047 & -20.66 & 261.6 & $\mathrm{~S} 0^{\mathrm{c}}$ & S0 & 2 \\
\hline MCG $5-27-13 \ldots \ldots \ldots \ldots$ & 7263 & -21.22 & 469.5 & $\mathrm{SBa}$ & $\mathrm{Sb}$ & 3 \\
\hline Fairall $316 \ldots \ldots \ldots \ldots$ & 4772 & -20.21 & 308.5 & S0 & S0 & 2 \\
\hline ESO $417-G 6 \ldots \ldots \ldots \ldots$ & 4792 & -19.88 & 309.8 & RS0 & $\ldots$ & 2 \\
\hline ESO $362-G 8 \ldots \ldots \ldots \ldots$ & 4616 & -20.44 & 298.4 & S0 & $\mathrm{Sa}$ & 1 \\
\hline \multicolumn{7}{|c|}{ Northern Sample ${ }^{d}$} \\
\hline Mrk $1 \ldots \ldots \ldots \ldots \ldots \ldots$ & 4970 & -19.66 & 321.3 & $\mathrm{~S}$ & $\mathrm{Sc}$ & 1 \\
\hline Mrk $3 \ldots \ldots \ldots \ldots \ldots \ldots$ & 4124 & -20.31 & 266.6 & S0 & S0 & 3 \\
\hline Mrk $34 \ldots \ldots \ldots \ldots \ldots \ldots$ & $15140^{\mathrm{c}}$ & $-21.36^{\mathrm{e}}$ & 978.6 & $\mathrm{~S}^{\mathrm{c}}$ & $\ldots$ & 3 \\
\hline Mrk $78 \ldots \ldots \ldots \ldots \ldots$ & 11288 & $-20.87^{e}$ & 729.7 & $\mathrm{SB}^{\mathrm{c}}$ & $\ldots$ & 1 \\
\hline Mrk $273 \ldots \ldots \ldots \ldots \ldots$ & 11390 & $-21.11^{\mathrm{e}}$ & 736.2 & Ring galaxy $^{\mathrm{c}}$ & $\ldots$ & 1 \\
\hline Mrk 463E ............... & 15209 & -22.5 & 983.1 & ? & $\ldots$ & 1 \\
\hline Mrk $477 \ldots \ldots \ldots \ldots \ldots$ & 11511 & -20.7 & 744.1 & $\mathrm{Sp}$ & $\ldots$ & 1 \\
\hline Mrk $533 \ldots \ldots \ldots \ldots \ldots$ & 8912 & -21.76 & 576.1 & Sbc & $\mathrm{S}(\mathrm{B}) \mathrm{c}$ & 1 \\
\hline Mrk $1066 \ldots \ldots \ldots \ldots \ldots$ & 3705 & -20.43 & 239.5 & SB0 & $\mathrm{Sc}$ & 1 \\
\hline Mrk 1073 ................. & 7097 & -21.98 & 458.8 & $\mathrm{SBb}$ & $\mathrm{Sc}$ & 1 \\
\hline NGC $1068 \ldots \ldots \ldots \ldots$ & 1144 & -21.45 & 73.95 & $\mathrm{Sb}$ & $\ldots$ & 3 \\
\hline NGC $2110 \ldots \ldots \ldots \ldots$ & 2153 & $-20.52^{\mathrm{e}}$ & 139.2 & SB0 & $\mathrm{Sa}$ & 2 \\
\hline NGC 5929 ............. & 2684 & $-20.17^{e}$ & 173.5 & $\mathrm{Sab}$ & So & 2 \\
\hline NGC $7212 \ldots \ldots \ldots \ldots$ & 7972 & -21.12 & 515.3 & $\mathrm{~S} ?$ & Irr? & 3 \\
\hline IC $3639 \ldots \ldots \ldots \ldots \ldots$ & 3137 & -20.4 & 202.8 & SBbc & $\mathrm{SBb}$ & 1 \\
\hline
\end{tabular}

Note.-Col. (1): Velocity in the galactic standard of rest from RC3; Col. (2): absolute blue magnitude using $B_{T}^{0}$ from RC3; Col. (3): scale in parsecs per arcsecond; Col. (4): Hubble type as in RC3; Col. (5): inner Hubble type from MGT; Col. (6): stellar population category.

${ }^{a}$ Stellar population categories are (1) young stellar population, (2) old stellar population, and (3) blue light of uncertain origin.

b Adopting distance to the Fornax cluster of $16.9 \mathrm{Mpc}$ (Tully 1988).

${ }^{c}$ From NED.

d Excluding galaxies in common with the southern sample.

${ }^{\text {e Using }} B_{T}^{0}$ from Whittle 1992.

levich (1990), Heckman et al. (1997), and González Delgado et al. (1998). In a recent work, Aretxaga et al. (2001) reported the detection of prominent Balmer absorption lines in six radio galaxies attributed to stars younger than $1 \mathrm{Gyr}$. Cid Fernandes \& Terlevich $(1992,1995)$ have shown how the presence of a nuclear starburst could solve the so-called FC2 problem (Tran 1995a, 1995b, 1995c), the problem of unpolarized blue light present in the spectra of many Seyfert 2 galaxies. Cid Fernandes, Storchi-Bergmann, \& Schmitt (1998), Storchi-Bergmann, Cid Fernandes, \& Schmitt (1998) and Schmitt, Storchi-Bergmann, \& Cid Fernandes (1999) showed that the FC2 problem in the optical can be solved if one takes into account the fact that the nuclear stellar population of Seyfert galaxies is varied and cannot in most cases be represented by an elliptical galaxy template. Intermediate-age $\left(\approx 10^{8} \mathrm{yr}\right)$ and young stars are responsible for the excess optical light in many cases.

Are nuclear starbursts ubiquitous in Seyfert 2 galaxies? In order to answer this question it is necessary to quantify the frequency of recent star formation episodes in or around Seyfert 2 nuclei. This has been done in two recent works by González Delgado, Heckman, \& Leitherer (2001; hereafter GD01) and Storchi-Bergmann et al. (2000; hereafter SB00), who have analyzed the near-UV spectra of 20 Seyfert 2 
galaxies each. Through spectral synthesis techniques, GD01 and SB00 have quantified the contribution of old, intermediate, and young stellar components to the spectra. Unambiguous signatures of recent star formation have been found in $50 \%$ of the galaxies of the sample of GD01, while SB00 have found such signatures in $30 \%$ of their sample. In another $30 \%$ of the galaxies of the two samples, a powerlaw component contributing less than $30 \%$ of the flux at $4020 \AA$ was necessary to reproduce the near-UV continuum. SB00 have shown that this component cannot be distinguished from the continuum produced by a starburst of $10 \mathrm{Myr}$ or younger for such small flux contributions. SB00 have called this component PL/YS (power law/young stars). If this latter component were entirely due to young stars, then the fraction of Seyfert 2 galaxies with recent star formation would increase to $80 \%$ for the northern sample and to $60 \%$ for the southern sample.

In order to investigate whether the incidence of recent star formation events is greater in the nuclei of Seyfert 2 galaxies than in non-Seyfert galaxies (implying a starburstAGN connection), it is necessary to compare the results obtained for the Seyfert galaxies with those for the nonSeyfert galaxies. This is the goal of this work. In $\S 2$ we describe the sample; in $\S 3$ we discuss the relation between the stellar population characteristics and the Hubble type of the galaxy; in $\S 4$ we compare the results for the Seyfert galaxies with those of non-Seyfert galaxies with the same Hubble type; in $\S 5$ we discuss the role of interactions; in $\S 6$ we discuss the relation between the stellar population and the inner morphology of the galaxy; and in $\S 7$ we present the conclusions of this work.

\section{SAMPLE}

We use for this work the combined samples of SB00 (hereafter the southern sample) and GD01 (hereafter the northern sample). Our goal is to collect a sample of Seyfert 2 galaxies in the local universe, spanning a range of morphological and environmental characteristics, whose spectra have been observed with similar instrumentation and for which the stellar population has been studied using similar techniques such that a common characterization of the stellar population can be used.

The southern sample comprises approximately $40 \%$ of the Seyfert 2 galaxies from the catalog of Veron-Cetty \& Veron (2000) with redshift $z<0.02$ and luminosity in the [O III] $5007 \AA$ emission line of $L_{[\mathrm{OIII}]}>10^{40} \mathrm{ergs} \mathrm{s}^{-1}$, which could be observed from the southern hemisphere. In addition, it contains the galaxies CGCG 420-015 and MCG 5-27-13, which obey the [O III] luminosity criterion but are somewhat more distant, with $z=0.029$ and 0.024 , respectively. The southern sample can be considered a local sample selected on the basis of the central source luminosity (via $L_{[\mathrm{OIII}]}$ ).

The northern sample was selected according to the flux of the central source: it comprises approximately $80 \%$ of the Seyfert 2 galaxies from the Whittle (1992) sample, observable from the northern hemisphere and with fluxes of $F_{\text {[OII] }}>0.6 \times 10^{-12}$ ergs $\mathrm{cm}^{-2} \mathrm{~s}^{-1}$ and/or $F_{1.4 \mathrm{GHz}}>80$ mJy. Besides five galaxies in common with the southern sample, the northern sample is composed of another seven galaxies that also obey the selection criteria of the southern sample, and in addition it contains eight galaxies with $z>0.02$ that have on average larger central source luminosities.
In summary, the combined sample contains 25 of the closest Seyfert 2 galaxies $(z<0.02)$ plus another 10 with $0.02<z<0.05$. The common selection criterion for all the galaxies is a luminosity of the central source higher than a lower limit, which produces $L_{[\mathrm{Om}]}>10^{40} \mathrm{ergs} \mathrm{s}^{-1}$. We will regard this sample as representative of nearby Seyfert 2 galaxies. As it was not selected by any property related to the stellar population, galaxy morphology, or environment, it is suitable to explore the relation among the latter three properties.

The properties of the galaxies relevant for this work are listed in Table 1, including radial velocities, absolute magnitudes (for $H_{0}=75 \mathrm{~km} \mathrm{~s}^{-1} \mathrm{Mpc}^{-1}$, used throughout this paper), scale at each galaxy, Hubble type as listed in the Third Reference Catalogue of Bright Galaxies (RC3; de Vaucouleurs et al. 1991) or in NASA/IPAC Extragalactic Database (NED), and an "inner" Hubble type proposed by Malkan, Gorjian, \& Tam (1998) on the basis of HST images (see discussion in $\S 5$ ). In the last column, we list a number representing a characterization of the stellar population at the nucleus based on the analysis of GD01 and SB00 as described below.

We have divided the stellar population into three categories characterized as follows: (1) the nuclear spectrum presents unambiguous signatures of recent star formation (younger than $500 \mathrm{Myr}$ ); these cases are also called composites (starburst + Seyfert), as their emission-line spectra have line ratios intermediate between those of starbursts and Seyfert galaxies (e.g., Cid Fernandes et al. 2001); (2) the stellar population is dominated by components older than 1 Gyr; (3) a PL/YS continuum is necessary to reproduce the spectra in the near-UV. The sample is made up of $15 \mathrm{com}$ posites $(\approx 40 \%$ of the sample), 10 dominated by an old stellar population $(\approx 30 \%)$, and $10(\approx 30 \%)$ for which there is a need for a blue continuum, which can be due to either a very young stellar population (but for which it is not possible to detect stellar absorption features) or a featureless continuum of nonstellar origin.

\section{HUBBLE TYPES}

We show in Figure 1 histograms of the Hubble types of our Seyfert 2 sample, where we have grouped the S0/a galaxies with the $\mathrm{Sa}$, the $\mathrm{Sab}$ with the $\mathrm{Sb}$, and the $\mathrm{Sbc}$ with the Sc. A few galaxies have uncertain Hubble types owing to either a distorted morphology or to the fact that the galaxies are too distant to allow a morphological classification based on available images. We have grouped the latter galaxies in Figure 1 in a column beyond that of Sc, identified as S?. The open histogram corresponds to the whole sample, and the hatched histograms to subsamples separated according to the stellar population categories described in $\S 2$ (from top to bottom: categories 1,3 , and 2).

From Figure 1 it can be concluded that the dominant Hubble types are $\mathrm{S} 0$ and $\mathrm{Sa}$, closely followed by $\mathrm{Sb}$, then the number of galaxies drops by more than $50 \%$ for the Sc, and then increases again for the uncertain types. In order to evaluate whether the morphological type distribution of our sample is representative of a better defined Seyfert sample, we compare it with that of Schmitt et al. (2001, hereafter S01). Their sample is selected on the basis of the $60 \mu \mathrm{m}$ infrared luminosity of the galaxies, a property believed to be isotropic, and contains approximately twice as many Seyfert 2 galaxies as our sample, with $18 \mathrm{~S} 0,18 \mathrm{Sa}, 14 \mathrm{Sb}$, $7 \mathrm{Sc}$, and two of uncertain type. Their distribution of 


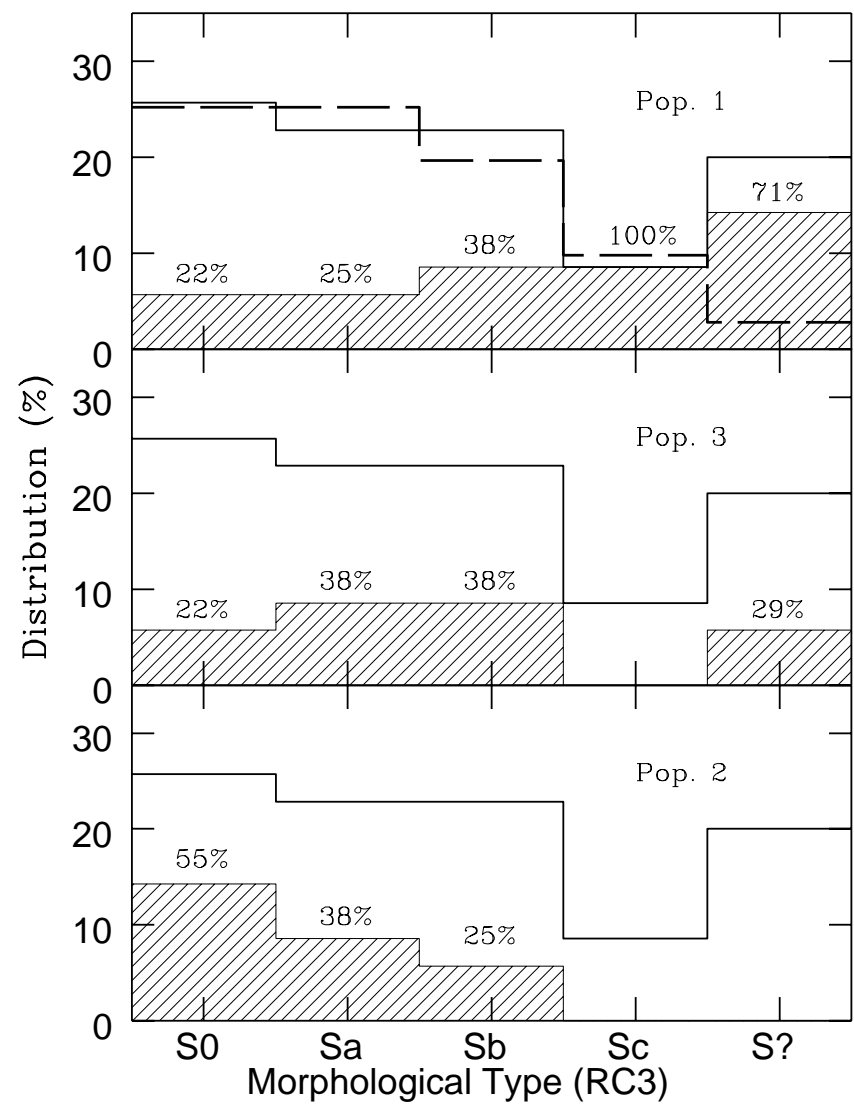

FIG. 1.-Open histograms: Percent distribution of Hubble types for the present Seyfert 2 sample (solid lines) compared with that of Schmitt et al. (2001) (dashed lines in top panel). Hatched histograms: Fractions of Seyfert 2 galaxies belonging to each stellar population category. From top to bottom, categories 1 (composites), 3 (PL/YS: blue continuum with uncertain origin), and 2 (old stellar population). The fractions are also labeled with corresponding percentages within each Hubble type.

Hubble types is also shown in Figure 1 as a dashed histogram. It is very similar to that of our sample, although it has a much smaller number of uncertain types. We attribute this difference to the fact that five of the seven $\mathrm{S}$ ? galaxies in our sample are the most distant ones, with much larger distances than those in the S01 sample, making them difficult to classify. Excluding these galaxies, the number of galaxies with uncertain classification in our sample is not significantly different from that in the S01 sample. As a result of

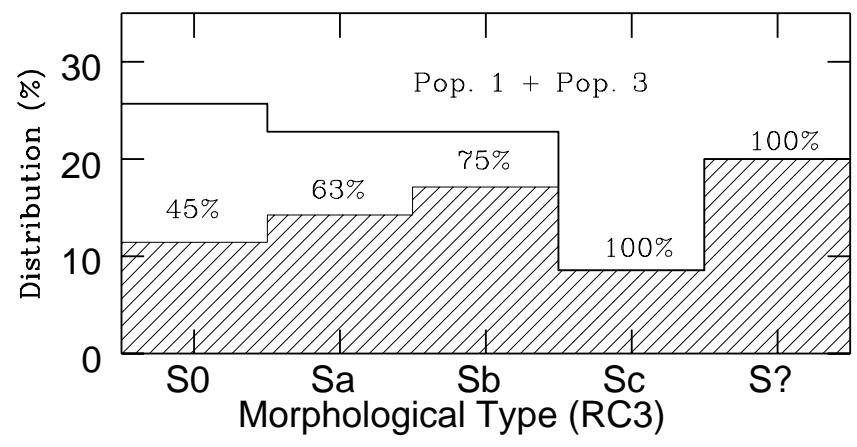

FIG. 2.-Open histogram shows the distribution of Hubble types as in Fig. 1, while the hatched histogram shows the fraction of galaxies for each Hubble type having a stellar population of category 1 or 3 . this difference, in Figure 1 we have normalized the S01 distribution to ours, excluding the uncertain types.

Regarding the stellar population category, the composites (category 1) comprise approximately $20 \%-25 \%$ of the $\mathrm{S} 0$ and $\mathrm{Sa}, \approx 40 \%$ of the $\mathrm{Sb}, 100 \%$ (although there are only three) of the Sc, and $\approx 70 \%$ of the $\mathrm{S}$ ?. The galaxies dominated by the old stellar population (category 2 ) are clearly concentrated toward the early types, with increasing percentages from $\approx 25 \%$ for the $\mathrm{Sb}$ to $\approx 60 \%$ for the $\mathrm{S} 0$. The galaxies with the PL/YS component (category 3) are approximately evenly distributed among the different Hubble types, comprising approximately $30 \%$ of the galaxies.

Now let us consider the possibility that the PL/YS continuum is produced by young stars. As discussed in Cid Fernandes et al. (2001), this could happen because of a contrast effect: the nuclear starburst would be too faint as compared with the contribution of the bulge, and the photospheric lines of the young stars would not be detected. The presence of a faint starburst in these cases would be detected only via the blue continuum and/or the dilution produced in the near-UV absorption features of the bulge stellar population as observed in the galaxies of category 3 . In order to take this possibility into account, we present in Figure 2 a revised version of the top panel of Figure 1, where we assume that all nuclei with stellar population of category 3 are also composites.

\section{COMPARISON WITH NON-SEYFERT SAMPLES}

A similar stellar population study to those of SB00 and GD01 was performed for local galaxies by Bica \& Alloin (1987) and Bica (1988, hereafter B88). We have used the B88 sample as a comparison sample for the distribution of the stellar population characteristics among the different Hubble types. The comparison is relevant because the stellar population characterization is essentially the same as that of our sample and the B88 sample is dominated by non-Seyfert nearby galaxies $(z<0.02)$. His goal in assembling the sample was to have a representative number of galaxies spanning Hubble types E-Sc and absolute magnitudes $-22<M_{B}<-16$. In addition, eight Seyfert galaxies and two starburst galaxies were also included. In order to compare our results with those of B88, we have excluded from his sample the latter and the elliptical galaxies. The remaining subsample comprises 117 spiral galaxies, distributed as $32 \mathrm{~S} 0,25 \mathrm{Sa}, 26 \mathrm{Sb}$, and $34 \mathrm{Sc}$ galaxies. B88 has avoided including in his study galaxies with uncertain morphology, so we cannot complete the $\mathrm{S}$ ? column with his results. The percent histogram of Hubble types of the B88 sample is shown in the top panel of Figure 3. On the basis of the stellar population analysis of B88, we were able to classify the stellar population of his work within the categories 1 and 2 described in $\S 2$. The corresponding distributions of stellar population categories are shown as hatched histograms in the first and third panels (from top to bottom) of Figure 3.

In order to check whether the distribution of morphological types in the B88 sample is representative of that of a "complete sample" of non-Seyfert galaxies, we have compared it with that of the Palomar spectroscopic survey of nearby galaxies by Ho, Filippenko, \& Sargent (1997, hereafter HFS97). This survey provides a representative sample of the galaxies in the near universe and can thus be used as a reference for the distribution of galaxies among the different 


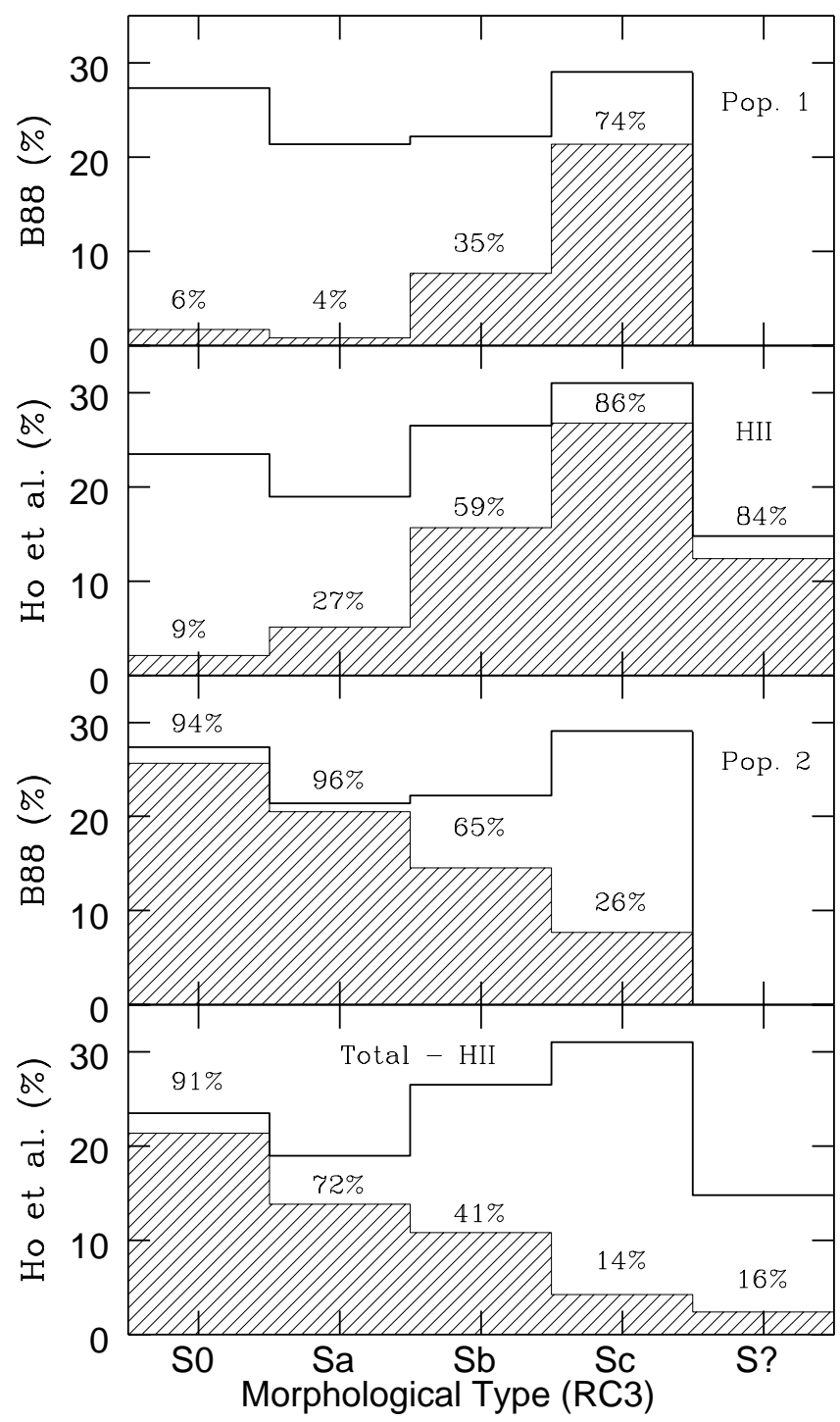

FIG. 3.-Open histograms: Percent distribution of Hubble types for two control samples of normal galaxies: the sample of Bica (B88) and that of Ho et al. (1997). Hatched histograms: Fraction of normal galaxies with recent star formation in the two top panels and with old stellar population in the two bottom panels. These fractions are also labeled with corresponding percentages within each Hubble type.

Hubble types. The HFS97 sample comprises most of the northern galaxies brighter than $B_{T}=12.5 \mathrm{mag}$, with a total of 486 galaxies, among which 57 are elliptical and 52 are Seyfert galaxies. The percent distribution of Hubble types of the HFS97 survey after excluding the elliptical and Seyfert galaxies is shown in the second panel (from top to bottom) of Figure 3. Since HFS97 include also galaxies with morphological types beyond $\mathrm{Sc}$, we construct the $\mathrm{S}$ ? bin, adding all galaxies with these later classifications. But in order to make the HFS97 distribution comparable to that of B88, we have normalized to the total number of galaxies from S0 to Sc, excluding the S?. HFS97 do not perform a stellar population study like B88 but have made a careful analysis of the emission-line spectra, classifying the galaxies as LINERs, Seyfert galaxies, and $\mathrm{H}$ II nuclei. Considering that an $\mathrm{H}$ II region spectrum is a tracer of very recent star formation, we tentatively use this classification as indicative of a stellar population of category 1 . The hatched histogram in the second panel (from top to bottom) of Figure 3 shows the distribution of the $\mathrm{H}$ II region nuclei within the Hubble types of the HFS97 sample.

Before comparing the Seyfert sample with the two above non-Seyfert samples, we also checked whether the three samples span similar ranges in absolute magnitude $M_{B_{T}^{o}}$. Figure 4 shows that this is indeed the case for the bulk of the galaxies in each sample. The distribution in absolute magnitude of the Seyfert galaxies is more similar to that of B88 than to that of HFS97, considering only the galaxies more luminous than $M_{B_{T}^{o}}=-19$, since that of HFS97 is systematically shifted relative to the distribution of Seyfert galaxies to less luminous galaxies by $\approx 0.5 \mathrm{mag}$. Below (lower luminosity) $M_{B_{T}^{o}}=-19$, there are low-luminosity tails in HFS97 and B88 distributions that are not present in the Seyfert distribution. However, these low-luminosity galaxies comprise only $12 \%$ of the B 88 and $17 \%$ of the HFS97 samples. We thus conclude that the Seyfert sample can be considered comparable to that of B88 in terms of absolute blue magnitude distribution, but both are shifted toward higher luminosity (by $0.5 \mathrm{mag}$ ) in comparison to the complete sample of HFS97.

From Figure 3 it can be observed that both the B88 and HFS97 surveys present an approximately uniform distribution of Hubble types along the sequence S0 to Sc and the number of galaxies with Hubble types later than Sc in the Palomar survey is approximately half the number of Sc galaxies.

A comparison between Figures 3 and 1 shows that the main difference between the Hubble type distributions of our Seyfert sample and those of the non-Seyfert samples is the smaller proportion of Scs among the Seyfert galaxies (approximately half that of the non-Seyfert galaxies). Another difference is the relative number of galaxies with uncertain or peculiar morphology, which is larger in the Seyfert sample when compared with that of the Palomar Survey.

Regarding the stellar population categories, the incidence of recent star formation in normal galaxies increases from

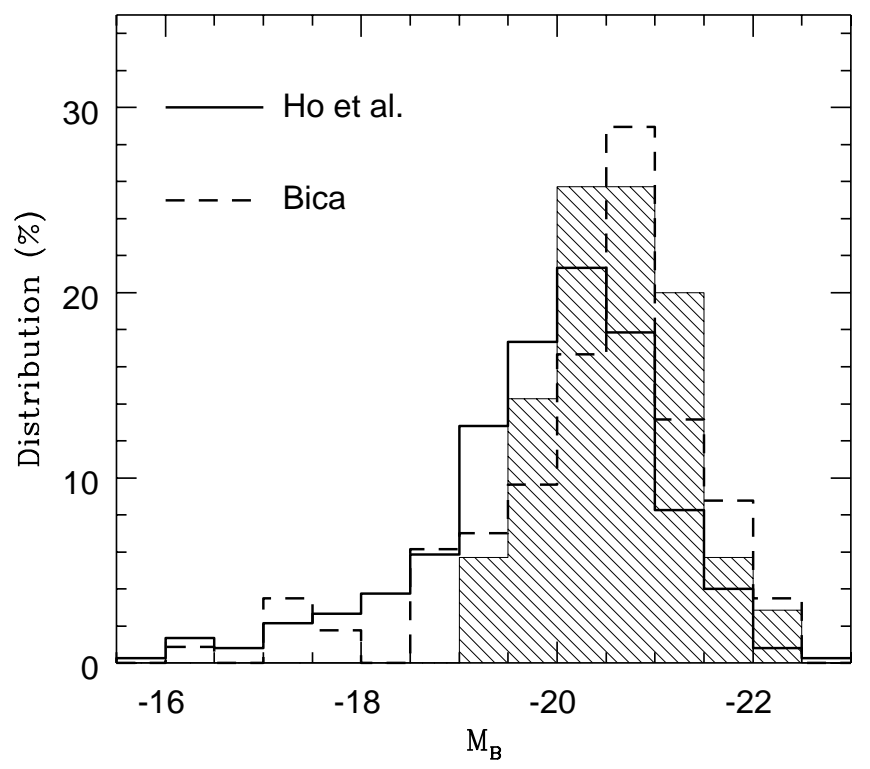

FIG. 4.- Hatched histogram: Distribution of absolute blue magnitudes of the Seyfert sample, which can be compared with that of B 88 (dashed lines) and Ho et al. (1997) (solid lines) samples. 
less than $10 \%$ for S0 to $70 \%-80 \%$ for Sc and S?. The incidence of recent star formation seems to be somewhat larger in the HFS97 sample than in the B88 sample. We attribute this difference to the distinct methods used to trace the young stellar population in the two samples, which favors the detection of fainter bursts of star formation when tracing them by the emission lines (HFS97), considering also that the detection limit of emission lines of HFS97 $(\approx 0.25 \AA)$ is lower than that of Bica's observations $(\approx 2 \AA$; Bonatto, Bica, \& Alloin 1989). For the Seyfert galaxies we identify a similar trend of increasing incidence of recent star formation toward later Hubble types. When compared with the B88 sample, the early-type Seyfert galaxies S0 and Sa present a larger incidence of recent star formation than the non-Seyfert galaxies, but in the case of $\mathrm{Sa}$, the incidence of star formation is similar to the percentage of $\mathrm{H}$ II nuclei in the HFS97 sample. Although the number of galaxies of the present sample is still small for a firm conclusion on this issue, a recent work by Raimann et al. (2001) has revealed two additional cases of nearby Seyfert 2 nuclei with recent star formation in S0 galaxies, supporting the conclusion that, at least for the S0 hosts, the Seyfert 2 nuclei show a greater incidence of star formation than those of the nonSeyfert galaxies.

We can also compare Figure 3 with Figure 2, in which we have assumed that the ambiguous blue continuum of category 3 is also due to young stars. If this is the case, it is clear that the incidence of recent star formation in Seyfert 2 galaxies would be larger than in non-Seyfert galaxies for all Hubble types. This conclusion would still hold even if only half of the population of category 3 is due to young stars. It is thus very important to investigate further the nature of the PL/YS continuum. Cid Fernandes et al. (2001), for example, have shown that for the same sample studied here, the galaxies show a continuum of infrared (IRAS) luminosities $L_{\mathrm{IR}}$, the more luminous being the composites (Seyfert $2+$ starburst). They have also found that $L_{\mathrm{IR}}$ is correlated with the fraction of the optical continuum due to blue light, the galaxies from category 2 being the less luminous in the infrared and showing almost no blue light, followed by the PL/YS cases up to the composites, which show larger fractions of blue light in the optical continuum and larger $L_{\mathrm{IR}}$. The Cid Fernandes et al. (2001) results thus suggest that the same phenomenon is occurring in the whole sample with a range of luminosities and that the PL/YS could indeed be due to young stars that are not detected because of the contrast effect discussed in $\S 4$.

The fraction of galaxies dominated by old stellar populations increases toward the early Hubble types in both Seyfert and non-Seyfert galaxies, although this fraction is smaller in the early-type Seyfert galaxies when compared to the non-Seyfert galaxies. This result is due to both the larger incidence of composites in S0 and Sa Seyfert galaxies discussed above and to the $\approx 30 \%$ incidence of galaxies with PL/YS continuum.

\section{GALAXY STRUCTURE IN THE INNER FEW KILOPARSECS}

Malkan et al. (1998, hereafter MGT) have recently published the results of an imaging survey of Seyfert and starburst galaxies using the Wide Field Planetary Camera 2 (WFPC2) aboard HST and the broadband filter F606W (which covers the spectral region $\approx 4700-7200 \AA$ ). Based on these images, which show a wealth of fine structure not detected in previous ground-based images, MGT have assigned a Hubble type for the inner region of each galaxy. This "inner Hubble type" is listed in column 6 of Table 1 . Ellipses identify the galaxies not observed in the imaging survey of MGT. Since these classifications are based on images obtained with the PC, which has a field of view of $37^{\prime \prime}$, they refer only to the inner few kiloparsecs of the galaxies except in the most distant cases.

We show in Figure 5 a histogram with the distribution of our sample according to the inner Hubble types of MGT (hereafter MGT type). This figure shows that many galaxies from our sample, in spite of having an early RC3 Hubble type, have a late MGT type. Interestingly, most cases of composites (category 1) have a late MGT type, indicating a relation between the MGT type of the Seyfert and its stellar population. A comparison between Figures 5 and 3 shows that the frequency of recent star formation in Seyfert 2 galaxies of Sc MGT type is remarkably similar to that in nonSeyfert galaxies of Hubble type Sc. The "Sc morphology" thus seems to be an indicator of the presence of recent star formation.

The dominant old population (category 2) is concentrated toward early MGT types, in this case in agreement with the relation with the large-scale Hubble type. The stellar population category 3 shows a similar behavior to category 2 .

The question we should now answer is what physical characteristics are behind the "Sc morphology" in F606W

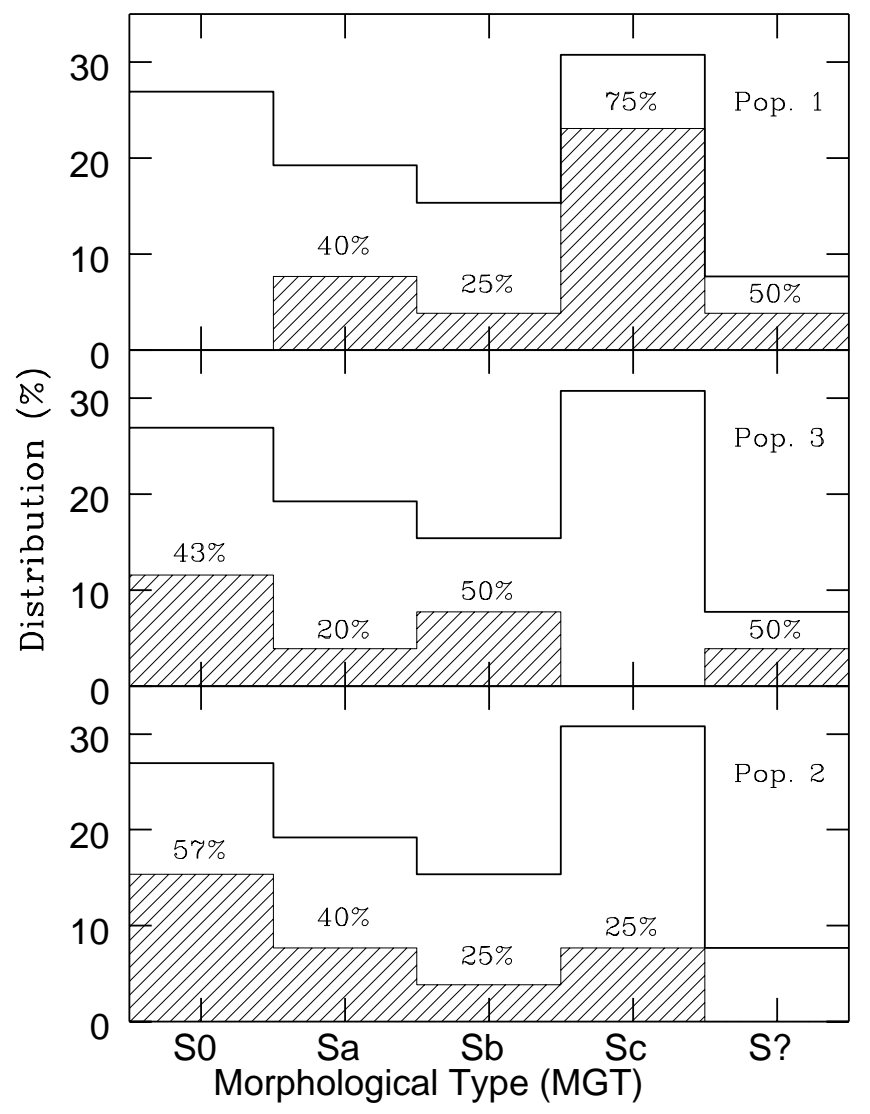

FIG. 5.-Open histograms show the distribution of MGT types ("inner Hubble types") of the Seyfert 2 sample, while the hatched histograms show the fraction of galaxies belonging to each stellar population category. From top to bottom: categories 1,3 , and 2 . The fractions are also labeled with corresponding percentages within each Hubble type. 
images? Such images are tracers of both the stellar distribution and dust. The dust distribution around Seyfert 2 nuclei has been recently studied by Martini \& Pogge (1999), combining F606W images with near-infrared NICMOS H images to construct $V-H$ color maps. These authors have shown that the nuclear regions of Seyfert 2 galaxies are very rich in gas and dust and have found nuclear spiral dust lanes on scales of a few hundred parsecs in 20 galaxies out of a sample of 24 Seyfert 2 galaxies. They suggest that these spiral dust lanes are the channels by which gas from the galaxy disks is being fed into the central engines. By comparing the $\mathrm{F} 606 \mathrm{~W}$ images with the $V-H$ maps of Martini \& Pogge, it can be concluded that the structure seen in the F606W images is mainly due to dust. In addition, as the F606W filter includes emission lines such as $\mathrm{H} \alpha-$ particularly strong in star-forming regions-large amounts of gas-emitting $\mathrm{H} \alpha$ may also contribute to the morphology of $\mathrm{F} 606 \mathrm{~W}$ images.

Based on the above, one possible interpretation for the late-type morphology of the inner regions of the Seyfert 2 galaxies with recent star formation is that these galaxies are particularly rich in emitting gas and associated dust, such that star formation can be triggered. A large amount of gas and dust is normally observed in galaxies with Hubble type Sc, which are known to be gas rich and to have a large incidence of nuclear starbursts. Thus it is not a surprise that the three galaxies of our sample with Hubble type Sc show recent star formation at the nucleus. What is new here is the case of the Seyfert 2 nuclei with earlier Hubble type hosts and recent star formation. These galaxies also seem to be particularly rich in gas in the inner few hundred parsecs around the nucleus to allow the triggering of star formation. This interpretation is supported by the results of the recent work of Cid Fernandes et al. (2001) for the same sample studied here, which have shown that the Seyfert 2 nuclei with clear signatures of recent star formation are the ones with larger far-infrared luminosities, consistent with stronger emission by starlight-heated dust in these galaxies.

\section{THE ROLE OF INTERACTIONS}

Having concluded that the Seyfert 2 nuclei with recent star formation are particularly rich in gas and dust in the inner regions, how is the gas transported to the central region of these galaxies? The problem of how the gas loses its angular momentum in this process has been discussed by many authors and several mechanisms have been suggested. One of these mechanisms is the interaction between galaxies (Gunn 1979; Hernquist 1989; Hernquist \& Mihos 1995), which can also trigger star formation before the nucleus is fed (Byrd et al. 1986; Byrd, Sundelius, \& Valtonen 1987; Lin, Pringle, \& Rees 1988). Large-scale bars can also remove angular momentum via gravitational torques, making the gas fall inward (Shlosman 1992). The latter mechanism seems to be particularly relevant in triggering nuclear starbursts, which preferentially occur in barred hosts (Heckman 1980; Balzano 1983; Kennicut 1994).

Structural distortions that could have been produced by interactions and bars have been indeed found by the pioneer work of Simkin, Su, \& Schwarz (1980). Dahari (1985) found an excess of companions in Seyfert galaxies when compared with a control sample of field galaxies, although the excess is small. This result has been recently confirmed by Rafanelli, Violato, \& Baruffolo (1995). Regarding bars, many recent studies (e.g., Mulchaey \&
Regan 1997) did not find more bars in Seyfert galaxies when compared with a control sample; the main structure they found is a nuclear spiral, in agreement with the work of Martini \& Pogge (1999) discussed in $\S 5$ and with the MGT classification. On the other hand, Knapen, Schlossman, \& Peletier (2000), making a careful match between the properties of a Seyfert sample with those of a comparison sample, do find a small excess of bars in Seyfert galaxies.

We do not find a relation between the circumnuclear stellar population category and the presence of a bar in the RCS classification. Nevertheless, for a conclusive result, a bar should be looked for in near-IR images (e.g., Knapen et al. 2000), which is beyond the scope of the present work.

We have checked the relation between interactions and the stellar population category in our sample by searching for companions around our galaxies using NED and the Digitized Sky Survey (DSS) plates and found obvious companions and/or signatures of interactions/mergers in 13 cases. The criteria used to identify companions included the proximity in the sky, similar radial velocity to that of the Seyfert galaxy, and apparent tidal distortions of the galaxy images. We list in Table 2 the 13 interacting galaxies together with the available information on the companion galaxies: name, distance from the Seyfert in kiloparsecs, radial velocity, and difference in absolute magnitude (magnitude of the companion minus that of the Seyfert). There are two cases of mergers in which it is not possible to separate the companion and four cases in which the Seyfert belongs to a group. In the latter cases, we have included in Table 2 the data for the galaxy from the group that is closest to the Seyfert.

There is only one case for which it was not possible to identify the companion: NGC 7130. In the DSS there seem to be two small companions: the one at $15.7 \mathrm{kpc}$ northwest listed in Table 2 plus another at $9.4 \mathrm{kpc}$ southwest (González Delgado et al. 1998). Shields \& Filippenko (1990) have obtained better quality images and report tidal distortion at faint light levels that could have been the result of interaction with the small galaxy at the northwest, which they also observe in their images. The other possible galaxy to the southwest is not observed. A spectrum of this small galaxy to the northwest would be necessary in order to conclude whether it is indeed gravitationally bound to NGC 7130.

For reference and comparison we also include in Table 2 the radial velocity and absolute magnitude of the corresponding Seyfert galaxy as well its stellar population category. All the above cases of interactions have been previously reported in the literature, and we thus list the corresponding references in the last column of Table 2. It can be observed from the Table that for most cases the available data, such as proximity to the Seyfert galaxy and similar radial velocity, support a physical association between the Seyfert galaxy and the companion. From Table 2 it can also be concluded that the companions are usually less luminous than the Seyfert galaxies, consistent with the theory by Hernquist \& Mihos (1995) in which minor mergers induce radial inflows that accumulate large quantities of interstellar gas in the nuclear regions of the host disks, which can then feed the nuclear black hole.

The proportion of 13 interacting galaxies out of a sample of 35 Seyfert 2 galaxies is similar to that of the larger Seyfert sample of S01, selected on the basis of the $60 \mu \mathrm{m}$ luminosity. Segregating the different stellar population categories, 
TABLE 2

SubSAMPle OF INTERACting SeYFERT GalaXies

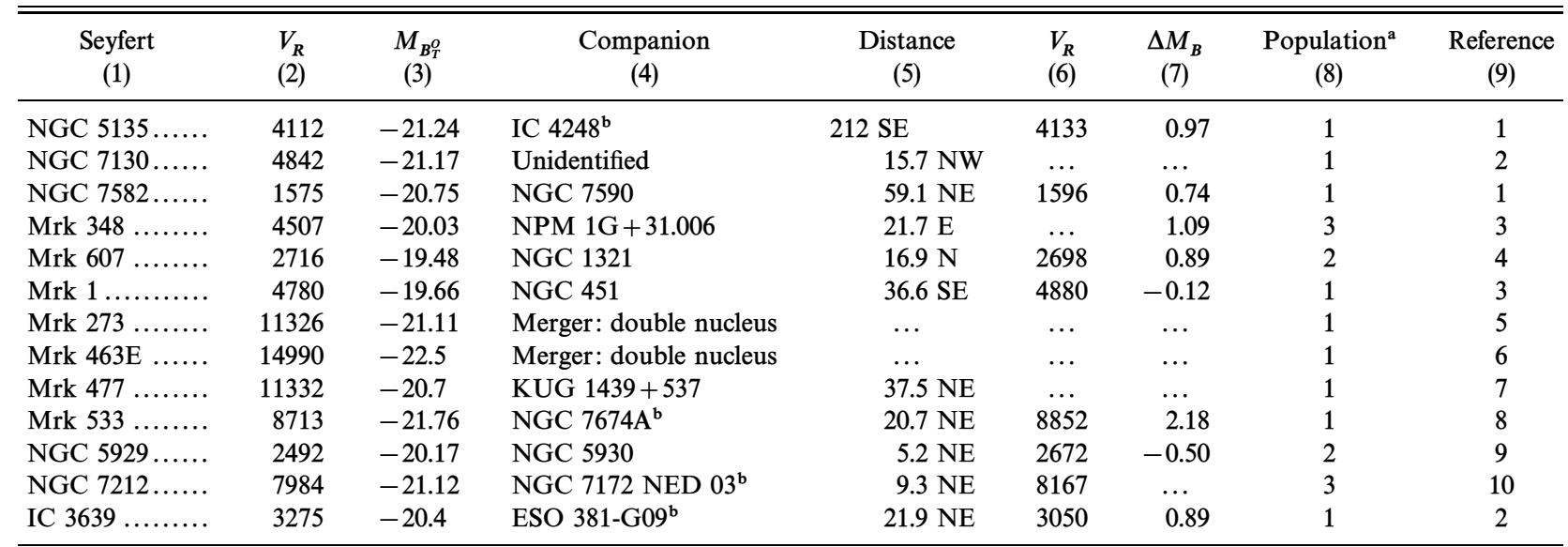

Note.-Columns: (1) Name of the Seyfert; (2) heliocentric velocity from NED; (3) $M_{B_{T}^{o}}$ as in Table 1 ; (4) name of the companion; (5) distance between Seyfert and companion in kpc using angular separation from NED; (6) heliocentric velocity of the companion from NED; (7) difference: magnitude of the companion minus that of the Seyfert from NED; (8) stellar population category of the Seyfert; (9) previous reference on the companion(s).

a Stellar population categories are (1) young stellar population, (2) old stellar population, and (3) blue light of uncertain origin.

b Belongs to a group; data are for the closest companion.

${ }^{c}$ In the DSS there seem to be two dwarf galaxies close to IC 5135; images by Shields \& Filippenko 1990 show tidal distortions and confirm the presence of the small unidentified galaxy to the northwest.

REFERENCES. - (1) Kollatschny \& Fricke 1989; (2) González Delgado et al. 1998; (3) Rafanelli et al. 1995; (4) Colbert et al. 1996; (5) Mazzarella \& Boroson 1993; (6) Heisler \& Vader 1994; (7) De Robertis 1987; (8) Verdes-Montenegro et al. 1997; (9) González Delgado et al. 1997; (10) Veilleux, Goodrich, \& Hill 1997.

among the 15 nuclei with recent star formation (category 1 or composites), nine have close companions. In comparison, of the 20 nuclei that do not show recent star formation, only four have close companions. Using another perspective, inspection of Table 2 shows that of the 13 galaxies with close companions or in groups, nine have recent nuclear star formation. This indicates a relation between the starburst activity and the presence of interactions. The overlap between the nuclei with recent star formation and the presence of companions can be observed in Figure 6, where we present their distributions in the histograms of Hubble types and MGT types.

Many observations have provided evidence for a causal link between strong nuclear starbursts and galaxy interactions, which is also consistent with theoretical predictions. The fraction of interactions in starburst galaxies ranges from $20 \%-30 \%$ for the lower luminosity starbursts up to $70 \%-95 \%$ for the higher luminosity ones (Kennicutt 1998 and references therein). The frequency of companions in our whole sample is $\sim 30 \%$ but increases to $\sim 60 \%$ when we consider the subsample of composites, which is close to that found among the most luminous starbursts.

The above result, combined with the evidence that the starbursts associated with interacting galaxies are also the youngest (Cid Fernandes et al. 2001; GD01; SB00), supports an evolutionary scenario for the relation between the nuclear starburst and the AGN as follows. First, galaxy interactions produce a flow of gas toward the center. When the amount of gas piled up in the nuclear region is large enough, this gas, besides feeding the active nucleus, triggers a starburst. The signatures of the interaction are still observable, the stellar population spectrum is of category 1 , and the emission-line ratios are intermediate between those of a Seyfert 2 galaxy and a starburst (Cid Fernandes et al. 2001). The starburst then fades, and the stellar population spectrum becomes dominated by older stars and is observed as one of category 2 or 3 with a Seyfert 2 emission-line spectrum (Cid Fernandes et al. 2001). This evolution is also observed in the MGT type, which changes from a late-type to an earlier type morphology.

The above scenario is also consistent with the results of numerical simulations of mergers (e.g., Hernquist \& Mihos 1995; Mihos \& Hernquist 1996). These simulations suggest that star formation at the nucleus begins $\approx 500-800 \mathrm{Myr}$ after the beginning of interaction (galaxies closer than a few

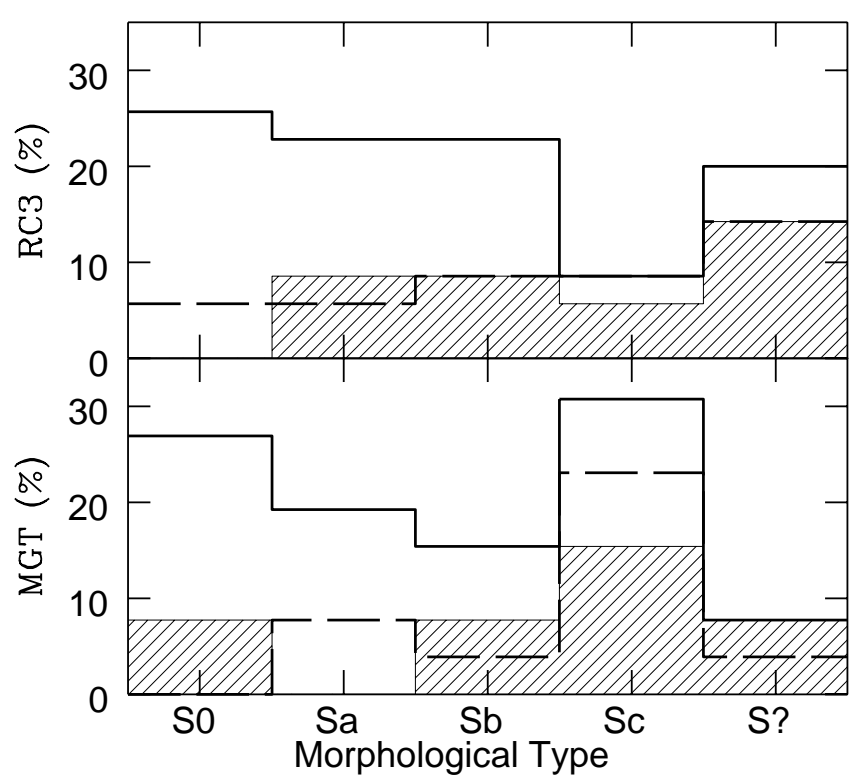

FIG. 6.-The histograms of Hubble types (top panel) and of the inner MGT types (bottom panel) for the Seyfert 2 sample, together with the fraction of galaxies with close companions (hatched histograms) and with recent circumnuclear star formation (dashed histograms). 
diameters), when clear signatures of the interaction are still visible. These signatures then almost disappear in another $200 \mathrm{Myr}$ or so, and only more subtle ones remain such as small distortions and rings, as those observed by Simkin et al. (1980) in Seyfert galaxies see also Hunt \& Malkan (1999).

A similar evolutionary scenario has been recently proposed by Lei et al. (2000) for LINERs. They have found that the intensity of AGN activity in LINERs increases with decreasing star formation contributions and suggest an evolutionary connection of LINERs with strong star formation and lower AGN activity to those with no star formation and stronger AGN activity.

\section{SUMMARY AND CONCLUDING REMARKS}

We have investigated the relation between the nuclear stellar population properties - in particular, the incidence of recent star formation - and both the galaxy morphology and the presence of companions in a sample of 35 Seyfert 2 galaxies. The results found for the Seyfert galaxies were compared with those of two control samples of non-Seyfert galaxies.

The main conclusions of this work are as follows:

The Hubble types of the Seyfert 2 galaxies are evenly distributed from $\mathrm{S} 0$ to $\mathrm{Sb}$, then the number of $\mathrm{Sc}$ galaxies drops to less than half the number of galaxies within each of the earlier Hubble types. When compared with a control sample of non-Seyfert galaxies, the present Seyfert 2 sample shows a $\sim 50 \%$ deficiency of Sc galaxies and an excess of galaxies with uncertain or peculiar morphology (although the latter result is apparently due in part to the difficulty in ascertaining a Hubble type to the most distant galaxies of the sample). This conclusion reinforces the known result that Seyfert nuclei are preferentially found in earlier type hosts (HFS97).

The number of Seyfert 2 galaxies with composite nuclei (Seyfert+starburst) increases toward the later Hubble types. The fraction of galaxies with recent star formation is similar to that found in non-Seyfert galaxies for the Hubble types $\mathrm{Sb}$ or later but seems to be larger in Seyfert 2 nuclei for the earlier Hubble types.

The nature of the ambiguous blue continuum PL/YS is a key issue in assessing the extent of the difference between the stellar population of Seyfert 2 nuclei and normal galaxies of the same Hubble type. If this continuum is in at least half the cases due to young stars, then the fraction of Seyfert 2 galaxies with recent circumnuclear star formation would be larger than that in normal galaxies for all Hubble types. This ambiguity can only be solved with high signalto-noise ratio UV or near-UV spectra obtained at high spatial resolution, observations which are presently feasible with $H S T$.

The number of Seyfert 2 nuclei dominated by an old stellar population increases toward the early Hubble types, similar to what is found in normal galaxies. Nevertheless, within each of the Hubble types S0, Sa, and Sb, the fraction of Seyfert 2 nuclei dominated by the old stellar population is systematically smaller than that in the normal galaxies owing to the larger fraction of Seyfert 2 galaxies with recent star formation and PL/YS continuum in these Hubble types.

There is a very good correlation between the presence of recent star formation and a late "inner Hubble type," assigned by MGT to the galaxies of our sample based on high spatial resolution HST F606W images. Our interpretation for this correlation is a larger gas content in the Seyfert 2 galaxies with recent star formation in and around the nucleus, which through the associated dust makes the gas distribution noticeable in the $\mathrm{F} 606 \mathrm{~W}$ images. This conclusion is consistent with the results of Cid Fernandes et al. (2001), who have found a correlation between the young stellar content and the infrared luminosity of the galaxy in the same sample, supporting a larger amount of dust emission in the Seyfert 2 galaxies with recent star formation.

Another good correlation is found between the presence of companions and the incidence of recent star formation in the Seyfert 2 nuclei. The frequency of companions in our whole sample is $30 \%$ but increases to $60 \%$ when we consider the subsample with recent star formation. Combined with the fact that the interacting galaxies are the ones with the youngest stellar population, this result suggests an evolutionary scenario in which the interaction is responsible for sending gas inward, which both feeds the AGN and triggers star formation, giving origin to a composite nucleus. The starburst then fades with time and the composite nucleus turns into a "pure" Seyfert 2 nucleus with an older stellar population. This scenario can reconcile the hypothesis that interactions are responsible for triggering nuclear activity in Seyfert galaxies with previous observational studies that do not find a large excess of interacting galaxies in Seyfert samples when compared with non-Seyfert ones. Signatures of the interactions are only clearly observed in the initial stages, which coincides with the phase in which a composite (Seyfert + starburst) nucleus is observed.

We are pleased to thank the hospitality of the INAOE, Tonantzintla, Mexico, and in particular Itziar Aretxaga and Daniel Kunth during the Guilhermo Haro workshop of 2000 July when this work was initiated. We also thank the suggestions by the referee, which helped to improve the paper. We acknowledge support from the Brazilian institutions CNPq, CAPES, and FAPERGS. We have made use of the NASA/IPAC Extragalactic Database, operated by the Jet Propulsion Lab, Caltech, under contract with NASA. The National Radio Astronomy Observatory is a facility of the National Science Foundation operated under cooperative agreement by Associated Universities, Inc.
Aretxaga, I., Terlevich, E., Terlevich, R. J., Cotter, G., \& Diaz, A. I. 2001, MNRAS, 325, 636

Bahcall, J. N., Kirharkos, S., Saxe, D. H., \& Schneider, D. P. 1997, ApJ, 479,642

Balzano, V. 1983, ApJ, 268, 602

Bica, E. 1988, A\&A, 195, 76

Bica, E., \& Alloin, D. 1987, A\&AS, 70, 281

Bonatto, C., Bica, E., \& Alloin, D. 1989, A\&A, 226, 23

Brotherton, M. S., et al. 1999, ApJ, 520, L87

Byrd, G. G., Sundelius, B., \& Valtonen, M. J. 1987, A\&A, 171, 16

Byrd, G. G., Valtonen, M. J., Valtaoja, L., \& Sundelius, B. 1986, A\&A, 166, 75

\section{REFERENCES}

Canalizo, G., \& Stockton, A. 2000, AJ, 120, 1750

Cid Fernandes, R., Heckmann, T., Schmitt, H. R., González Delgado, R., \& Storchi-Bergmann, T. 2001, ApJ, in press

Cid Fernandes, Storchi-Bergmann, T., \& Schmitt, H. R. 1998, MNRAS, 297, 579

Cid Fernandes, R., \& Terlevich, R. 1992, in ASP Conf. Ser. 31, Relationships between Active Galactic Nuclei and Starbursts Galaxies, ed. A. V. Filippenko (San Francisco: ASP), 241

. 1995, MNRAS, 272, 423

Colbert, E. J. M., et al. 1996, ApJS, 105, 75

Collin, S., \& Zahn, J. P. 1999, A\&A, 344, 433

Dahari, O. 1985, ApJS, 57, 643 
De Robertis, M. M. 1987, ApJ, 316, 597

de Vaucouleurs, G., de Vaucouleurs, A., Corwin, H. G., Jr., Buta, R. J., Paturel, G., \& Fouqué, P. 1991, Third Reference Catalogue of Bright Galaxies (Berlin: Springer) (RC3)

Ferrarese, L., \& Merrit, D. 2000, ApJ, 539, L9

Gebhardt, K., et al. 2000, ApJ, 539, L13

González Delgado, R., Heckman, T., \& Leitherer, C. 2001, ApJ, 546, 845

González Delgado, R., Perez, H., Tadhunter, C., Vilchez, J., \& RodriguezEspinosa, J. M. 1997, ApJS, 108, 155

González Delgado, R., et al. 1998, ApJ, 505, 174

Gunn, J. 1979, in Active Galactic Nuclei, ed. C. Hazard \& S. Mitton (Cambridge: Cambridge Univ. Press), 213

Heckman, T. M. 1980, A\&A, 88, 365

Heckman, T. M., et al. 1997, ApJ, 482, 114

Heisler, C. A., \& Vader, P. 1994, AJ, 107, 35

Hernquist, L. 1989, Nature, 340, 687

Hernquist, L., \& Mihos, J. C. 1995, ApJ, 448, 41

Ho, L. 1999, in Observational Evidence for Black Holes in the Universe, ed. S. K. Chakabarti (Dordrecht: Kluwer), 157

Ho, L. C., Filippenko, A. V., \& Sargent, W. L. W. 1997, ApJ, 487, 579

Hunt, L. K., \& Malkan, M. A. 1999, ApJ, 516, 660

Kennicutt, R. C. 1994, in Mass-Transfer Induced Activity in Galaxies, ed.

I. Shlosman (New York: Cambridge Univ. Press), 131

Kennicutt, R. C., Jr. 1998, ARA\&A, 36, 189

Knapen, J. H., Schlossman, I., \& Peletier, R. F. 2000, ApJ, 529, 93

Kollatschny, W., \& Fricke, K. J. 1989, A\&A, 219, 34

Lei, S. J., Huang, J. H., Zheng, W., Ji, L., \& Gu, Q. S. 2000, ApJ, 544, L31

Lin, D. N. C., Pringle, J. E., \& Rees, M. J. 1988, ApJ, 328, 103

Magorrian, J., et al. 1998, AJ, 115, 2285

Malkan, M. A., Glorjian, V., \& Tam, R. 1998, ApJS, 117, 25

Martini, P., \& Pogge, R. W. 1999, AJ, 118, 2646

Mazzarella, J. M., \& Boronson, T. A. 1993, ApJS, 85, 27
Mihos, J. C., \& Hernquist, L. 1996, ApJ, 464, 641

Mulchaey, J. S., \& Regan, M. 1997, ApJ, 482, L135

Norman, C., \& Scoville, N. 1988, ApJ, 332, 124

Perry, J. J., \& Dyson, J. E. 1985, MNRAS, 213, 665

Rafanelli, P., Violato, M., \& Baruffolo, A. 1995, AJ, 109, 1546

Raimann, D., Storchi-Bergmann, T., Bica, E., \& Alloin, D. 2001, MNRAS, 324,1087

Schmitt, H. R., Antonucci, R. R. J., Ulvestad, J., Kinney, A. L., Clarke, C. J., \& Pringle, J. E. 2001, ApJ, 555, 663

Schmitt, H. R., Storchi-Bergmann, T., \& Cid Fernandes, R. 1999, MNRAS, 304,35

Shields, J. C., \& Filippenko, A. V. 1990, AJ, 100, 1034

Shlosman, I. 1992, in ASP Conf. Ser. 31, Relationships between Active Galactic Nuclei and Starburst Galaxies, ed. A. V. Filippenko (San Francisco: ASP), 335

Simkin, S. M., Su, H. J., \& Schwarz, M. P. 1980, ApJ, 237, 404

Storchi-Bergmann, T., Cid Fernandes, R., \& Schmitt, H. R. 1998, ApJ, 501, 94

Storchi-Bergmann, T., Raimann, D. I., Bica, E., \& Fraquelli, H. A. 2000, ApJ, 544, 747

Terlevich, E., Diaz, A. I., \& Terlevich, R. 1990, MNRAS, 242, 271

Tran, H. D. 1995a, ApJ, 440, 565 $1995 \mathrm{~b}, \mathrm{ApJ}, 440,578$

$1995 \mathrm{c}$, ApJ, 440, 597

Tully, R. B. 1988, Nearby Galaxies Catalog (Cambridge: Cambridge Univ. Press)

Veilleux, S., Goodrich, R. W., \& Hill, G. J. 1997, ApJ, 477, 631

Verdes-Montenegro, L., del Olmo, A., Perea, J., Athanassoula, E., Marquez, I., \& Augarde, R. 1997, A\&A, 321, 409

Veron-Cetty, M. P., \& Veron, P. 2000, Catalog of Quasars and Active Galactic Nuclei (9th ed.; Garching: ESO), 1

Whittle, M. 1992, ApJS, 79, 49 